\title{
Radiology in paediatric non-traumatic thoracic emergencies
}

\author{
Charlotte de Lange
}

Received: 26 November 2010 / Revised: 7 April 2011 / Accepted: 14 June 2011 /Published online: 6 July 2011

(C) European Society of Radiology 2011

\begin{abstract}
Non-traumatic thoracic emergencies in children are very frequent, and they usually present with breathing difficulties. Associated symptoms may be feeding or swallowing problems or less specific general symptoms such as fever, sepsis or chest pain. The emergencies always require a rapid diagnosis to establish a medical or surgical intervention plan, and radiological imaging often plays a key role. Correct interpretation of the radiological findings is of great importance in diagnosing and monitoring the illness and in avoiding serious complications. Plain radiography with fluoroscopy still remains the most important and frequently used tool to gain information on acute pulmonary problems. Ultrasound is the first choice for the detection and treatment of simple and complicated pleural effusions. Cross-sectional techniques such as multidetector computed tomography (MDCT) and magnetic resonance imaging (MRI) are mainly used to study pulmonary/mediastinal masses and congenital abnormalities of the great vessels and the lungs. This article will discuss the choice of imaging technique, the urgency of radiological management and the imaging characteristics of acquired and congenital causes of non-traumatic thoracic emergencies. They represent common conditions involving the respiratory tract, chest wall and the oesophagus, as well as the less frequent causes such as tumours and manifestations of congenital malformations.
\end{abstract}

Keywords Emergencies $\cdot$ Radiology $\cdot$ Paediatric $\cdot$ Thorax

C. de Lange $(\square)$

Department of Diagnostic Imaging and Intervention, Pediatric section, Oslo University Hospital, Rikshospitalet, P.O. box 4950 Nydalen,

0424 Oslo, Norway

e-mail: charlotte.eva.delange@oslo-universitetssykehus.no

\section{Introduction}

Thoracic non-traumatic emergencies are the most common emergencies in children, and they may be very dramatic, especially in cases of acute respiratory failure. Radiology is essential in making a rapid diagnosis in daily clinical practice in order to plan treatment and to avoid the severe complications of the disease. Radiologists should be able to recognise the large spectrum of thoracic emergencies in children and their differences depending on the age of the child. When choosing the appropriate technique for investigation, the consequences of radiation exposure in children as well as the clinical indication and patient age must be considered. Knowledge of the different radiological methods, when to use them and at what age is therefore very important. The objective of this article is to review radiological findings in different non-traumatic thoracic emergencies and to discuss how the clinical presentation influences the choice of radiological method.

\section{Physiology, anatomy and pathological conditions specific to children}

Knowledge of the development of the lungs and airways in children is helpful for understanding the specific findings and presentation in acute emergencies. The collateral pathways of ventilation in the lungs (channels of Lambert and pores of Kohn) are underdeveloped until the age of approximately 8 years. In addition children have more mucus production in the bronchial tree than adults $[1,2]$. The smaller calibre of their airways combined with softer walls may lead to earlier and more symptomatic airflow disturbances, even with minor amounts of oedema and mucus. This can be illustrated by an example considering a 
thickening of the tracheal mucosa of $1 \mathrm{~mm}$ : In an adult with a tracheal diameter of $20 \mathrm{~mm}$ this 1-mm thickening causes a reduction of the tracheal lumen of $17.5 \%$. In a baby with a tracheal diameter of $5 \mathrm{~mm}$ the same thickening of $1 \mathrm{~mm}$ causes a lumen reduction of $63.8 \%$. Narrowing of the tracheal lumen by infectious oedema or extrinsic mechanical obstruction may cause an acute check-valve mechanism of the airways causing air trapping. In infants there is also a closer relationship between the thoracic organs, and pathological processes in the thoracic cavity may have an earlier and more severe impact on the neighbouring organs. The thymus is a soft tissue mass that does not compress the airways or the vessels in the anterior mediastinum. The presence (of a thymic shadow) is normal until the age of $4-5$ years and does not cause any clinical symptoms. It is a well-known pitfall and should not be mistaken for a mediastinal tumour.

In general, the smaller the child the more urgent the need for diagnosis and treatment in a thoracic emergency because small children may decompensate rapidly with haemodynamic and respiratory failure.

\section{Clinical presentation}

The clinical presentation of thoracic emergencies can be variable but breathing difficulties of all kinds are the most common: stridor, cough, wheezing, respiratory distress, tachypnoea, nasal flaring and intercostal retractions. Wheezing is an abnormal, mainly expiratory, sound due to intrathoracic obstruction. Expiratory stridor is due to narrowing of the small airways causing air trapping in the lungs while inspiratory stridor is a sign of supraglottic narrowing. Due to the embryological origin of the airways and oesophagus both arising from the foregut, swallowing problems or even haemoptysis can be associated, especially in neonates and infants [3]. General symptoms such as fever, sepsis, chest pain and failure to thrive may often be associated.

\section{Radiological imaging}

Radiation safety must always be considered when imaging children. Compared with adults, children have an increased sensitivity to irradiation to their growing body. Their young age and longer lifetime expectancy increase the risk of developing radiation-induced cancer. When imaging is needed to establish a diagnosis, it is important to follow the ALARA principle (as low as reasonably achievable) meaning that the imaging technique with no or the lowest irradiation needed to achieve the right diagnosis should always be chosen. When ionising techniques are considered necessary, dose-reducing equipment and protocols for paediatric examinations are mandatory, following established international guidelines [4-6]. For radiography/ fluoroscopy this means for example using automatic exposure control and pre-filter exchange, the lowest pulse frequency possible, radiation-free adjustment of object positioning, removable grids, the option to store fluoroscopic images and no exposure unless necessary. CT examinations of the thorax should always be performed with adjusted imaging parameters for children. The tube voltage should be $80-100 \mathrm{kVp}$ rather than $120 \mathrm{kVp}$, and automatic dose modulation software should be used. In addition, in-plane breast shields reduce the radiation to breast tissue considerably without affecting image quality. One acquisition limited to the area of interest is in most cases sufficient [7].

The appropriate radiological method for the initial work-up of acute respiratory failure is plain radiography with inspiratory and sometimes expiratory films or fluoroscopy, and this still remains the most important and frequently used tool for gaining information. Fluoroscopy can be used to evaluate the motion of the mediastinum, diaphragm and airways but for evaluation of diaphragmatic motion, ultrasound is preferred as an easy, non-irradiating alternative suitable for bedside use. Dynamic examinations of the oesophagus or trachea using contrast media are sometimes needed. Ultrasound is the first choice for the diagnosis and treatment of pleural and pericardial effusions. Cross-sectional techniques such as multidetector computed tomography (MDCT) and magnetic resonance imaging (MRI) are mainly used for the study of pulmonary parenchymal and mediastinal masses including congenital anomalies of the lungs. Advanced imaging techniques in computed tomography (CT) and MRI may only occasionally be required in the acute situation.

When cross-sectional imaging is needed in the emergency, clinically acute setting in our institution, we tend to use CT before MRI in smaller children in spite of the exposure to radiation. MDCT has a spatial resolution superior to MRI, and there is generally no need for sedation or general anaesthesia.

Scintigraphy and positron emission tomographycomputed tomography (PET-CT) are not commonly used in the emergency situation but may be complementary in studying and staging some types of tumour such as lymphoma. Ultimately, the choice depends on the clinical presentation, the patient's age and the available radiological equipment at hand and with the ALARA principle in mind.

In the follow-up and monitoring of thoracic emergencies, chest radiography and ultrasound still play a central role, while cross-sectional imaging gives a better overview of the pathological features. Advanced techniques such as dynamic airway CT, CT or MR angiography (CTA, MRA) and cine MR imaging may be valuable in providing relevant vascular and functional information. 


\section{Thoracic emergencies in different age groups}

The spectrum of thoracic emergencies in children relative to age is shown in Table 1. Viral infections and foreign bodies dominate among infants with a maximum incidence between 6 months and 3 years. Tumours such as teratoma and neuroblastoma typically present in infants, while leukaemia and lymphoma are seen in children and adolescents. Congenital abnormalities are often diagnosed antenatally or after birth and in infancy, but may become symptomatic later on. Normal findings for different age groups may mimic pathological processes. During infancy, the thymus may present as an anterior mediastinal mass with various forms and shapes and must not be mistaken for a tumour or infection.

\section{The newborn period}

In neonates, the chest radiograph and ultrasound are the most common tools used to diagnose medical disease in premature and term babies and to monitor their treatment. Frontal view is usually sufficient, with lateral films only sometimes necessary in semi-urgent situations, e.g. to confirm the presence or location of a pneumothorax/ pneumomediastinum. CT or high-resolution computed tomography (HRCT) is seldom required for causes such as air leaks or pulmonary infection. When congenital anomalies of the airways or pulmonary masses are present, cross-sectional imaging combined with dynamic methods is often needed. This review will mainly focus on diseases that occur in term newborn babies.

\section{Air leaks}

Pneumothorax is mainly associated with mechanical ventilation in premature babies, but spontaneous pneumothorax may be seen in term babies. A tension pneumothorax with deviation of mediastinal structures may be fatal especially if not recognised and treated immediately [3, 8]. Air may spread into the mediastinum/pleural cavity with lateral or medial pneumothorax and even into the pericardial cavity, subcutaneous tissue or downwards into the peritoneal cavity. Chest radiography is the first choice of imaging in the urgent and semi-urgent situation. A lateral film with horizontal beam with the child in a lateral or supine decubitus position may be required to confirm the diagnosis.

Pneumomediastinum in term babies without treatment with mechanical ventilation may occur but is infrequent [9]. Usually moderate clinical symptoms associated with typical radiological findings are found with the thymus surrounded by air in the mediastinum - an "angel wing" or "spinnaker sail" sign. This type of air leak should resolve spontaneously, although it can also progress and cause subcutaneous emphysema in the neck and dissect into the peritoneal cavity (Fig. 1a-c).

\section{Neonatal pulmonary infections}

Respiratory infections in neonates and children are extremely common for reasons explained earlier, but neonatal pneumonia in developed countries is now relatively rare. The infections may occur in utero, during passage through the birth canal, or just after birth. Prolonged rupture of membranes, maternal vaginal and placental infection, contamination of the infant from maternal faecal material or foetal sepsis are predisposing factors. Bacterial infections are more frequent than viral infections in neonatal pneumonia and include nonhaemolytic streptococcus, staphylococcus and Escherichia coli and other Gram-negative and Gram-positive organisms. Viral infections include organisms such as adenovirus, herpes simplex, influenza and parainfluenza. Respiratory syncytial (RS) virus can be acquired from infected siblings and/or healthcare workers and is especially common in the winter season. Congenital tuberculosis and fungal infec-

Table 1 Thoracic emergencies in children of different ages

\begin{tabular}{|c|c|c|c|c|c|}
\hline Pathology & $\begin{array}{l}\text { Neonate } \\
\text { (0-1 month) }\end{array}$ & $\begin{array}{l}\text { Infant } \\
\text { (1-23 months) }\end{array}$ & $\begin{array}{l}\text { Preschool child } \\
\text { ( } 2-5 \text { years })\end{array}$ & $\begin{array}{l}\text { Child } \\
\text { (6-12 years) }\end{array}$ & $\begin{array}{l}\text { Adolescent } \\
\text { (13-18 years) }\end{array}$ \\
\hline Airleaks & Yes & Yes & & & Yes, spontaneous \\
\hline Infection & Viral bronchiolitis & Viral/bacterial pneumonia & $\begin{array}{l}\text { Bacterial } \\
\text { pneumonia }\end{array}$ & $\begin{array}{l}\text { Bacterial } \\
\text { pneumonia }\end{array}$ & \\
\hline Foreign body & & Yes, 6 months -3 years & Yes, 6 months -3 years & & \\
\hline Tumour & & Teratoma, neuroblastoma & $\begin{array}{l}\text { Lymphoma, } \\
\text { leukemia }\end{array}$ & $\begin{array}{l}\text { Lymphoma, } \\
\text { leukemia }\end{array}$ & $\begin{array}{l}\text { Lymphoma, } \\
\text { leukemia }\end{array}$ \\
\hline $\begin{array}{l}\text { Congenital } \\
\text { anomalies }\end{array}$ & $\begin{array}{l}\text { Heart, lung, vascular } \\
\text { and tracheobronchial } \\
\text { anomalies; diaphagmatic } \\
\text { hernia }\end{array}$ & $\begin{array}{l}\text { Heart, lung, vascular } \\
\text { and tracheobronchial } \\
\text { anomalies; diaphagmatic } \\
\text { hernia }\end{array}$ & & & \\
\hline
\end{tabular}


Fig. 1 a-d Pneumothorax. a Tension pneumothorax in a premature baby on the right side with a mediastinal shift to the left and compression of the left lung. b Anteroposterior and $\mathbf{c}$ lateral view of pneumomediastinum with air around the thymus - the "angel wing sign" - and bilateral basal pneumothorax in a term baby with respiratory problems. d A 7-year-old girl with pneumothorax on the right side and air leak into the mediastinum and subcutaneous tissue on the neck and axillae bilaterally
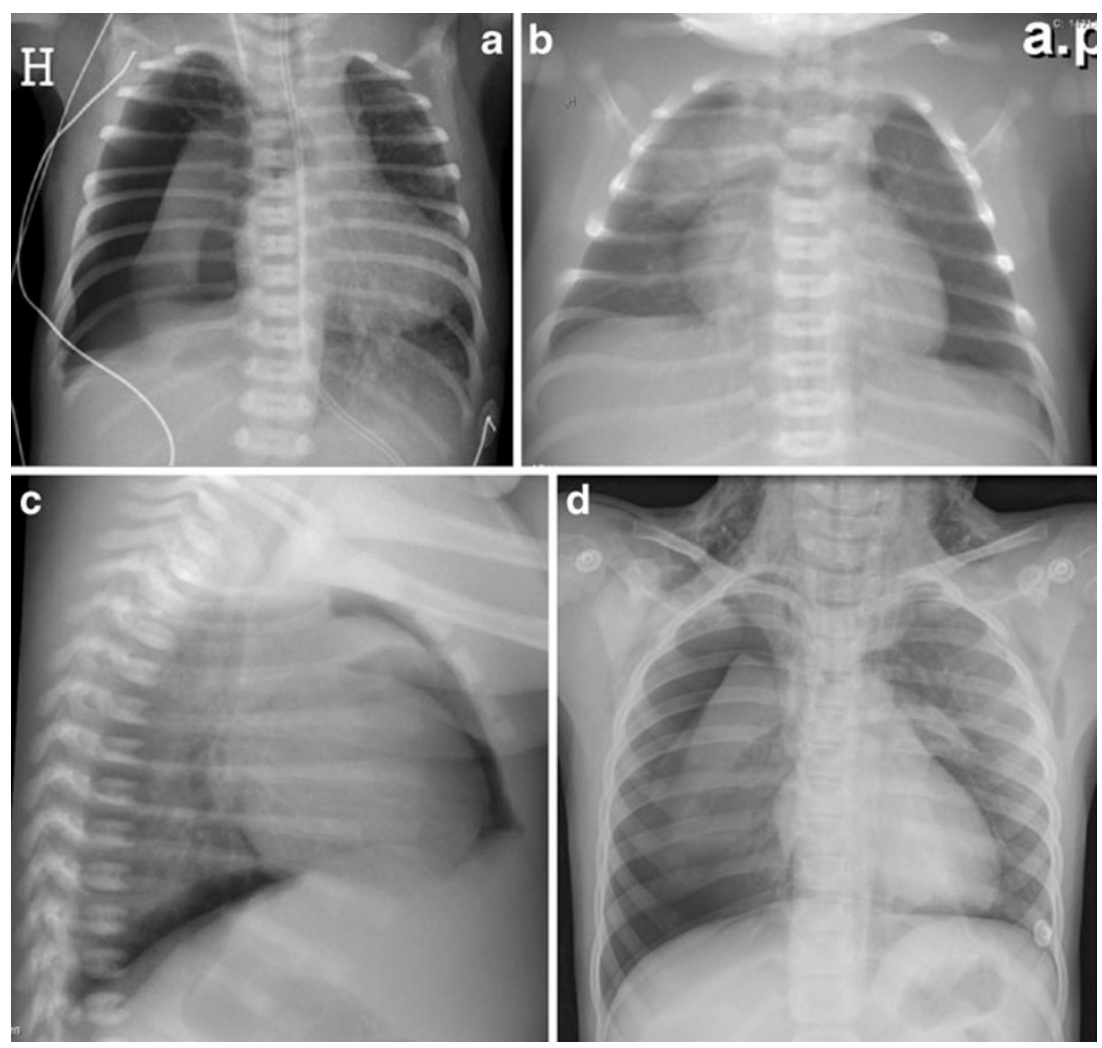

tions are rare. Clinical symptoms are often cough, respiratory distress, fever of variable degree or even sepsis, but may also be non-specific such as malaise or irritability [10]. The typical radiological findings in a viral infection include perihilar linear opacities, hyperinflation with air trapping and atelectasis (Fig. 2). Bacterial infections may present as alveolar densities. Like viral infections they may also have non-specific findings or a pattern resembling wet lung disease or respiratory distress syndrome (RDS). It is often difficult to differentiate between bacterial and viral infections on the basis of clinical and radiological findings, and radiography is performed as a part of the initial evaluation to determine if pneumonia is present or not.

\section{Tracheobronchial anomalies}

Pathological conditions of the central airways are more commonly detected in children than in adults. Diseases involving the trachea can easily be missed in children unless the airways are carefully studied. Congenital anomalies of the airways such as an aberrant tracheal bronchus or tracheal stenosis may often be combined with congenital heart disease or be a part of congenital syndromes. They can cause acute respiratory problems during pulmonary infections often in the neonatal period. Tracheal anomalies may be seen on plain radiography, but MDCT with multiplanar reconstructions (MPR) and volume-rendering (VR) is superior in depicting the anatomy
(Fig. 3). A contrast-enhanced CT will be able to detect an associated congenital heart anomaly and/or heterotaxy syndromes. For dynamic evaluation of the degree of stenosis and malacia (softening of the tracheobronchial wall causing expiratory collapse), bronchoscopy is used. Bronchography can be valuable in selected cases as a supplement to bronchoscopy and MDCT to clarify anatomy and function. The radiation exposure must be considered, but it remains a simple safe method of obtaining images of the major airways [11-13]. Dynamic CT of the airways with modern MDCT systems with radiation dose-reducing

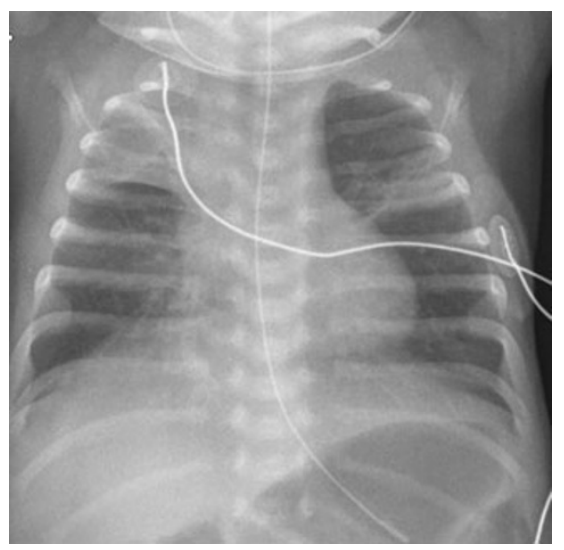

Fig. 2 A neonate with respiratory syncytial (RS) virus infection. Chest radiography shows hyperinflation with air trapping and atelectasis 
Fig. 3 a-c A 1-year-old boy with a viral infection and severe breathing problems. a Chest radiography findings raised the suspicion of the narrowing of the distal trachea. b Axial CT and c volumerendering reconstruction reveal an aberrant right upper bronchus and a long distal tracheal stenosis caused by complete cartilage rings
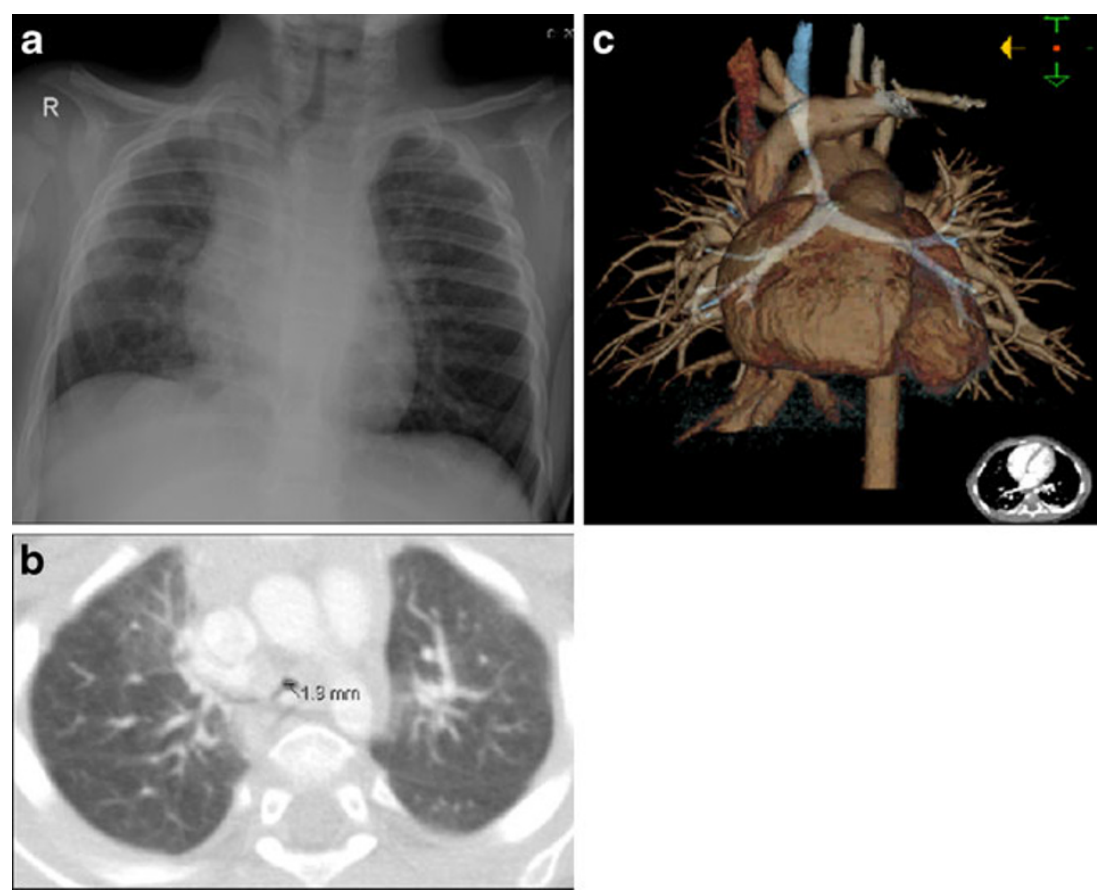

techniques might be a non-invasive alternative to bronchography in the future [14].

\section{Congenital diaphragmatic hernia}

Congenital diaphragmatic hernia $(\mathrm{CDH})$ is a rare lifethreatening and complex condition involving the diaphragm, but it also affects the lung and pulmonary vasculature. The hernias may be anterior (through the foramen of Morgagni) or posterior (through the foramen of Bochdalek), where the latter is the most frequently occurring [15]. Most hernias are diagnosed antenatally with ultrasound or MRI, but late presentation of CDH may occur [16, 17]. Depending on the size and position of the diaphragmatic defect, bowel loops, stomach and even solid abdominal organs may herniate into the thoracic cavity. Initial chest radiography postnatally shows an opacified hemithorax with mass effect and a contralateral shift of the mediastinum. Once air is introduced into the gastrointestinal tract, air-filled cystic figures corresponding to bowel loops and the stomach are seen in the thorax and gas-filled bowel in the abdomen may be lacking. Deviation and displacement of catheters and nasogastric tubes are seen in many patients, and an intrathoracic placement of a nasogastric tube is highly suggestive of CDH. An important differential diagnosis to $\mathrm{CDH}$ is congenital cystic adenomatoid malformation (CCAM)/bronchopulmonary sequestration (BPS) and primary pulmonary agenesis or hypoplasia. These conditions are often diagnosed antenatally. Chest radiography and ultrasound of the abdomen/diaphragm are the examinations of choice to confirm the diagnosis of
CDH. Sometimes careful inflation of air or contrast material via the nasogastric tube in search of bowel peristalsis can be necessary. In cases of late presentation the defect in the diaphragm is often small and the thorax will slowly fill up with bowel loops (Fig. 4) [15, 18]. The outcome of these patients is mainly related to the degree of pulmonary hypoplasia on the affected side, the presence of pulmonary hypertension and the need for extra-corporeal membrane oxygenation (ECMO) in the neonatal period $[15,17,18]$.

\section{Beyond the neonatal period}

\section{Air leaks in children/adolescents}

In older children, pneumothorax is usually caused by penetrating trauma, cystic fibrosis, asthma, pulmonary infection or foreign body aspiration with air trapping. In adolescents, spontaneous pneumothorax may occur (Fig. 1d). In some cases an anteroposterior view in expiratory phase may be necessary to visualise a minor pneumothorax.

\section{Pulmonary infections}

In infants, viral infections are more frequent than bacterial infections, and they may cause pneumonia. Clinical symptoms are often cough, respiratory distress and fever of variable degree, but they may also be non-specific such as malaise, irritability, headache and thoracic or abdominal pain. The chest radiography in two planes is the first choice 


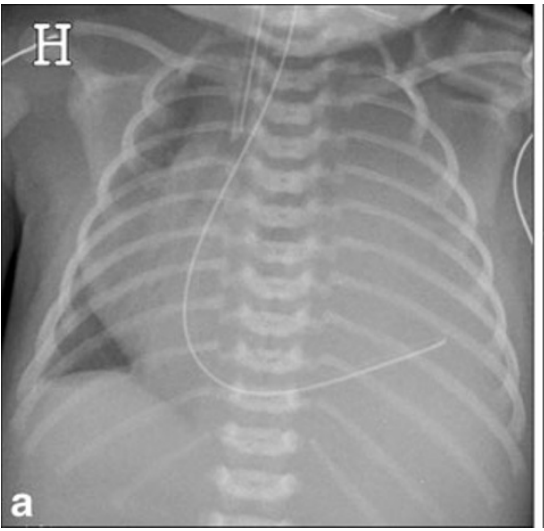

Fig. 4 a, b Congenital diaphragmatic hernia (CDH). a Antenatally diagnosed $\mathrm{CDH}$ in a neonate where chest radiography immediately after birth reveals opacity of the left thorax, mediastinal shift and the nasogastric tube directed into the left thorax. b A neonate presenting

of imaging in the acute situation and often sufficient for clinical management.

Findings in viral infections are related to their location in the airways, where inflammation and necrosis lead to peribronchial oedema, presenting as bilateral perihilar linear opacities, peribronchial markings, hyperinflation and air trapping (Fig. 5). However 30\% of patients have normal findings at chest radiography [19]. Peribronchial markings are subjective findings and represent a spectrum ranging from near normal to grossly abnormal [1]. The finding of prominent hila on the lateral view can help in determining whether the markings are increased. Hyperinflation in small children is often easier to diagnose on lateral films showing a flattened diaphragm and increased anterior-posterior diameter. Both air trapping and sub-segmental atelectasis are very frequent in association with viral infections in the lower respiratory tract.

Bacterial infections are seen more frequently around and after 5 years of age. They are typically unilateral and located in the peripheral alveoli causing lobar opacities.

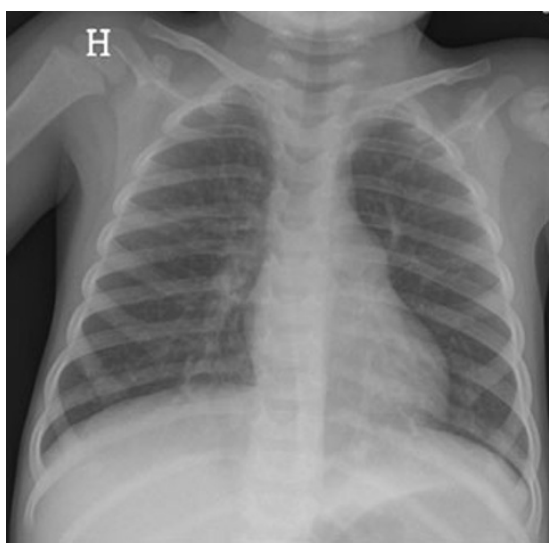

Fig. 5 Chest radiography of a 3-year-old girl reveals a generalised hyperinflation with bilateral perihilar linear opacities

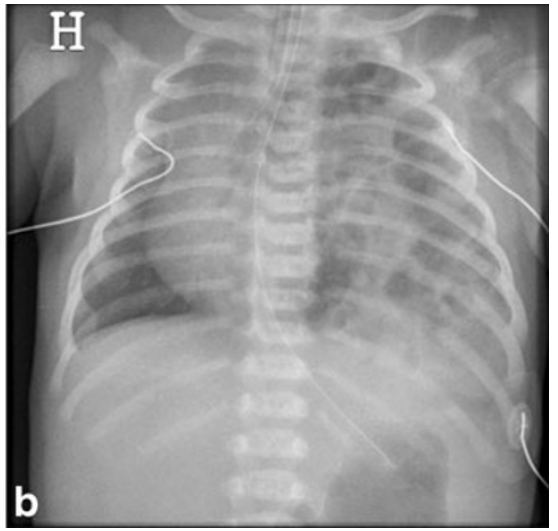

with respiratory distress on the fifth day of life. $\mathrm{CDH}$ with air-filled structures in the left thorax causing mediastinal shift to the right. The nasogastric tube is in the ventricle in a normal sub-diaphragmatic position

This corresponds to true air space opacification caused by alveoli filled with pus (Fig. 6). The most frequent location is in the lower lobes, and it is not uncommon that pneumonia is recognised in a lower lobe on the abdominal radiograph obtained for abdominal pain. Bacterial pneumonia is commonly associated with parapneumonic effusion and empyema. Overlapping imaging findings between viral and bacterial infections may occur and radiology can only suggest the aetiology in typical findings. One example is mycoplasma pneumonia, which represents $30 \%$ of lower respiratory tract infections in school-aged children and can have a "viral" or "bacterial" radiographic pattern [1]. Round pneumonia is a special entity presenting in children less than 8 years of age (with undeveloped collateral ventilation). The typical radiographic sign is a round mass, and follow-up radiography after treatment is warranted to exclude other entities such as tumour or a bronchogenic cyst [20].

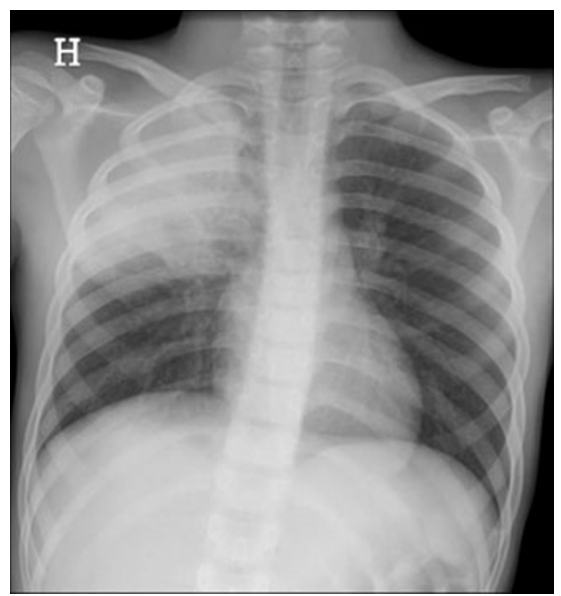

Fig. 6 Chest radiography of a 9-year-old boy. Lobar opacity without volume reduction in the right upper lobe 
The value of performing chest radiography in a child with respiratory infection is debated. Many authors agree that chest radiograph should be obtained in febrile infants only when signs of respiratory distress are present [21] and there is evidence that radiological findings will change treatment $[1,22]$.

\section{Complications to pulmonary infection: parapneumonic effusion/empyema}

Bacterial pneumonia develop adjacent pleural effusion in $40 \%$ of cases, but only $10-15 \%$ become infected and require drainage [23]. However, pleural empyema in children is increasing in incidence in several countries because of improved diagnosis but also the reduction in the number of patients treated with antibiotics [24, 25]. The term empyema is used to describe the presence of pus (macroscopic) in the pleural cavity. The causing pathogens and the outcome in children with empyema differ from those in adults [24], and the distinction between a complicated or a non-complicated pulmonary pleural effusion (PPE) may be difficult. Chest radiography is always performed as the first examination and can often suggest but not definitively establish the presence of empyema. An anteroposterior view often reveals a consolidation of a lower lobe and a parapneumonic collection with a medial convex border, indicating a loculated effusion. A large effusion may cause an almost complete opacification of the affected hemithorax. Often there is an ipsilateral concave scoliosis as the child is avoiding movement of the affected hemi-thorax. Ultrasound should always be carried out to confirm the presence and size of an effusion and to establish the presence of fibrinous septations. Ultrasound is usually sufficient in characterising the effusion for clinical management, and the use of additional radiographic views such as decubitus or combined erect and supine views is generally unnecessary.

To predict the stage of the pathophysiological process of the effusion (from exudative to fibrinopurulent and organised) is difficult with ultrasound. Contrast-enhanced CT can demonstrate the location of the effusion and often typical enhancement of the thickened parietal pleura. CT will not be able to show septations and, like ultrasound, cannot predict the viscosity of the effusion, and lack of enhancement of the pleura does not exclude the presence of an infected effusion [26]. CT should not be performed routinely in the diagnosis of a PPE but should be reserved for more complicated cases where the combination of ultrasound and chest radiography fails to distinguish a loculated empyema from an intraparenchymal abscess or a cavitary necrosis/necrotising pneumonia (Fig. 7). Opinions differ with regard to how aggressive the management of parapneumonic effusions should be. Antibiotics, thoracocentesis/chest drain with intrapleural fibrinolysis in combination with antibiotics, surgical thoracotomy with decortication and video-assisted thoracoscopic surgery are
Fig. 7 a-c A boy, 6 years of age, presenting with high fever and cough. a Chest radiography with consolidation of the left lower lobe. There is a typical ipsilateral scoliosis and a pleural effusion with medial convexity indicating a loculated effusion. b Ultrasound confirms the presence of an effusion with fibrinous septations traversing the pleural space. c Axial contrast-enhanced CT shows the pleural effusion and enhancing parietal pleura (white arrow)
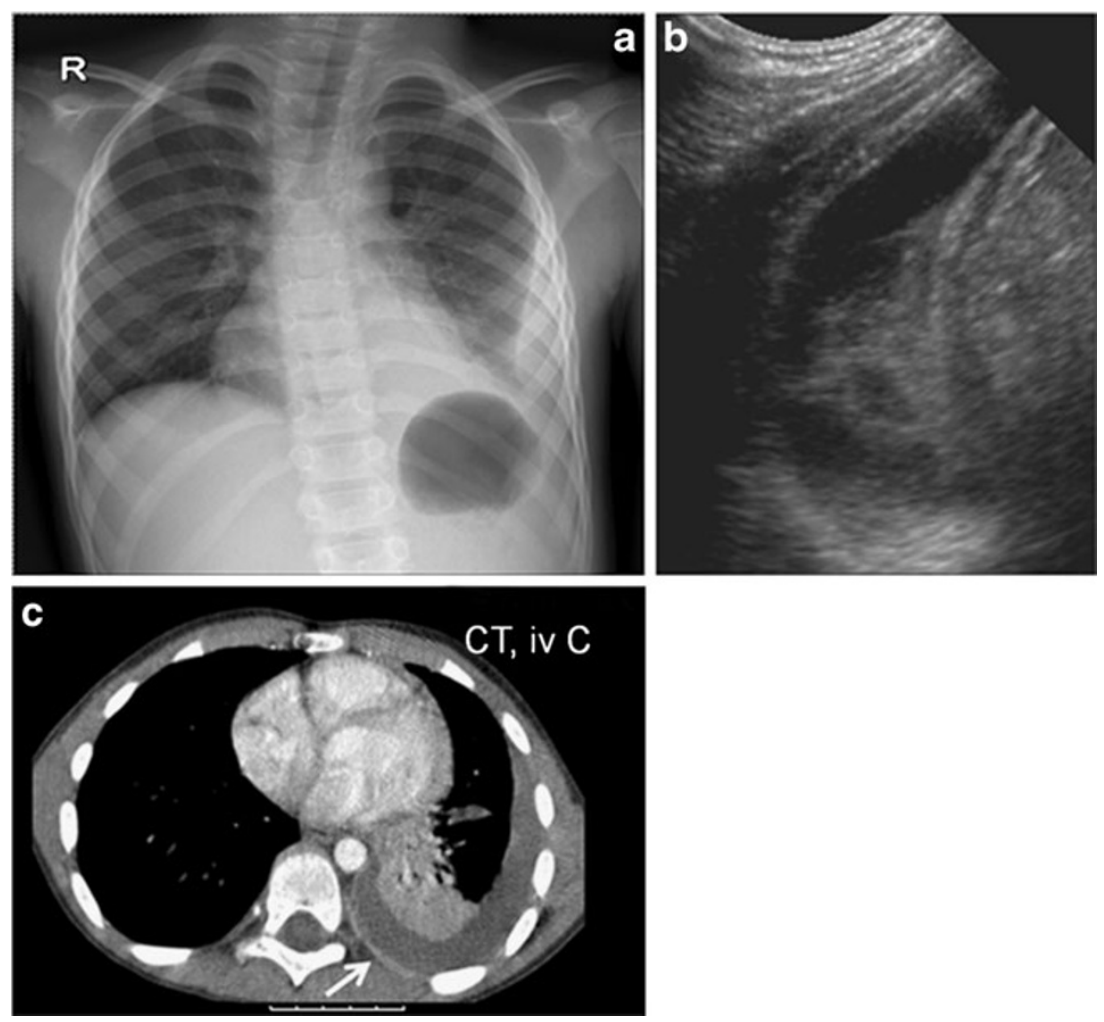
the optional treatments [24]. The choice of treatment differs among hospitals, and the decision of management should not be based on imaging characteristics alone. There is a consensus that drainage is required in cases of enlarging effusions despite antibiotics or compromised respiration [25, 27]. If a chest drain is used to evacuate the effusion, this should be placed under ultrasound guidance. Additional fibrinolytic treatment may be indicated when the aspect of the effusion on ultrasound is loculated or echogenic [24, 27].

In the follow-up, ultrasound and chest radiography are preferred. In complicated cases with lack of response to treatment with remaining sepsis and effusion, contrastenhanced CT can be required to identify the position of drains, loculations and parenchymal complications especially in immunocompromised children. Preoperative CT before thoracotomy is often demanded by surgeons. In many cases CT is performed revealing a failed tube drainage needing surgical treatment.

\section{Complications of pulmonary infection: parenchymal complications}

Suppurative lung parenchymal complications represent varieties of lung abnormalities ranging from abscess to cavitary necrosis with bronchopleural fistulas. Chest radiography and ultrasound are often not sufficient in the diagnosis of these complications where $\mathrm{CT}$ is more sensitive and may be necessary to detect and establish the extent of the complication. However, some of these parenchymal complications have a favourable prognosis in children, and a careful consideration of the impact on clinical management of a CT examination is warranted, keeping in mind the issue of radiation dose [26]. A lung abscess is defined as a fluid/gas collection within the lung parenchyma with a well-defined border and enhancing rim. A true lung abscess that requires treatment with drainage is uncommon in otherwise healthy children [1]. Cavitary necrosis represents a complication of severe pneumonia where necrosis of a consolidated lobe is presented with a variable number of thin-walled cysts. It is the most common parenchymal complication of pneumonia and is usually associated with staphylococcal infection or Streptococcus pneumoniae [26]. The CT appearance is of decreased contrast enhancement and loss of normal lung architecture with the multiple thin-walled cavities filled with fluid and air (from the bronchial communication; Fig. 8). In a non-complicated pneumonia with atelectasis on the other hand, there is diffuse contrast enhancement. Differentiating underlying congenital lesions may be difficult, and serial follow-up examinations may be needed. Purulent pericarditis, although very rare in immunocompetent children, is also a possible complication that may require urgent evacuation.

\section{Foreign body aspiration/ingestion}

Inhalation of a foreign body is one of the most common household accidents and is most frequent in the youngest
Fig. 8 a-d Cavitary necrosis in a neonate with Ebsteins anomaly and complications in the postoperative period after heart surgery. a Chest radiography shows a longstanding opacity of the entire left lung with gradual development of a large air bubble in the lower part. There were clinical signs of bacterial infection. b Ultrasound reveals a round, wellcircumscribed, sub-pleural lesion containing air and fluid. c Contrast-enhanced CT in an axial plane shows an air fluid level and no enhancement of the peripheral rim and adjacent lung. d Chest radiography after ultrasound-guided drainage of the necrosis and bronchopleural fistula
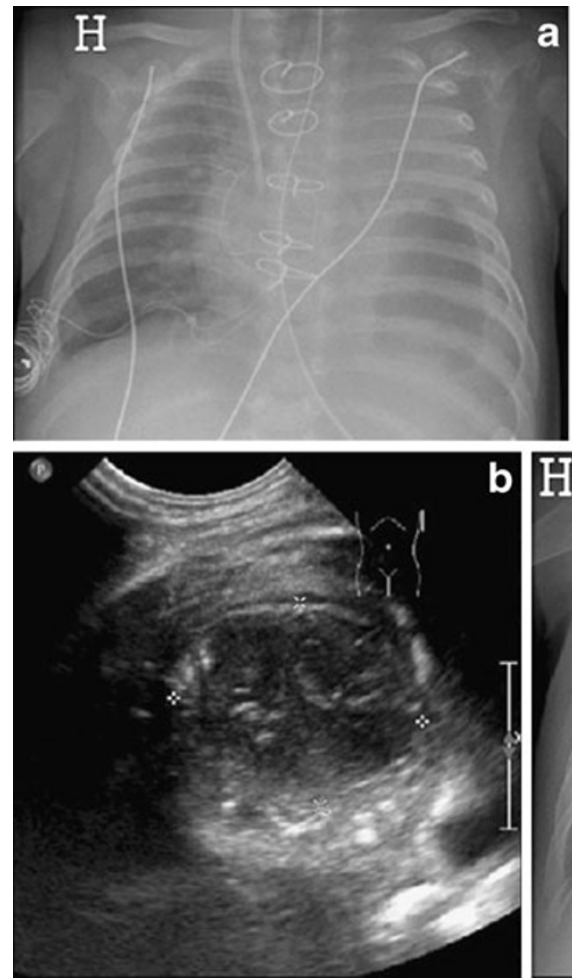

b $\mathrm{H}$ c
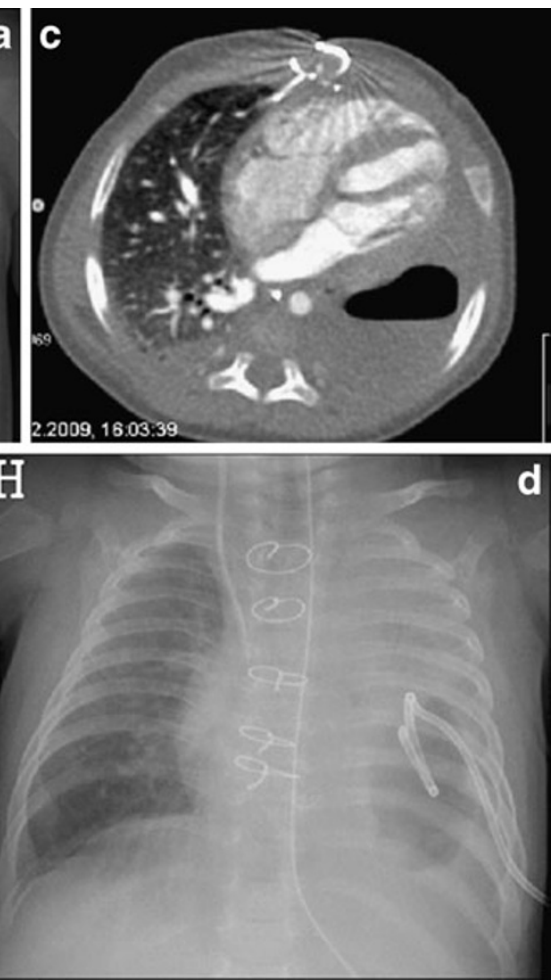
children with a peak frequency between 6 months and 3 years $[3,20,28]$. Although the course is most frequently benign, severe complications or even death may develop if there is an acute and total obstruction of the larynx or trachea. It is an important differential diagnosis in a child presenting with acute stridor or wheezing. Organic bodies, such as vegetables, are the most frequent foreign bodies inhaled, while coins are the most frequently swallowed. In children foreign bodies are usually inhaled into the stem bronchi with no predilection for the right or left main bronchus [29]. When a foreign body is suspected, chest radiography with lateral view, expiratory films and fluoroscopy may be helpful, although there is a high rate of negative radiological findings (sensitivity $70 \%$, specificity $62 \%)[3,28]$. The additional lateral view may help to further localise the object to the oesophagus or airway. The observation of obstructive emphysema on chest radiography and with fluoroscopy, a contralateral mediastinal shift and ipsilateral paradox or restricted diaphragmatic motion during breathing supports the diagnosis of check-valve air trapping due to an endobronchial foreign body (Fig. 9). Bronchoscopy can confirm the diagnosis, and the foreign body can often be removed at the same time (Fig. 10). Lack of and delayed treatment may cause recurrent pneumonia and development of bronchiectasis requiring lobectomy [3, 30]. The sequelae are more frequent and important with late diagnoses, and some authors report a complication rate of $60 \%$ [30]. An ingested foreign body may cause respiratory as well as swallowing problems (Fig. 11), and radiography with fluoroscopy, possibly with upper GI series, can help in confirming and differentiating the location in the trachea or oesophagus.

Multidetector CT is reserved for more complicated cases or late diagnosis requiring a more thorough depiction of additional pathological features in order to plan treatment.

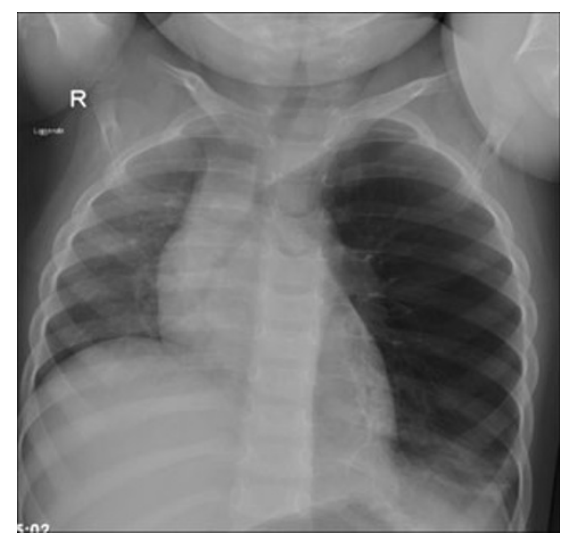

Fig. 9 Foreign body aspiration in a 2-year-old girl with a 2-week history of coughing and wheezing. Chest radiography shows obstructive emphysema of the left lung with a mediastinal shift supporting the suspicion of a foreign body in the left main bronchus. A walnut fragment was removed during bronchoscopy
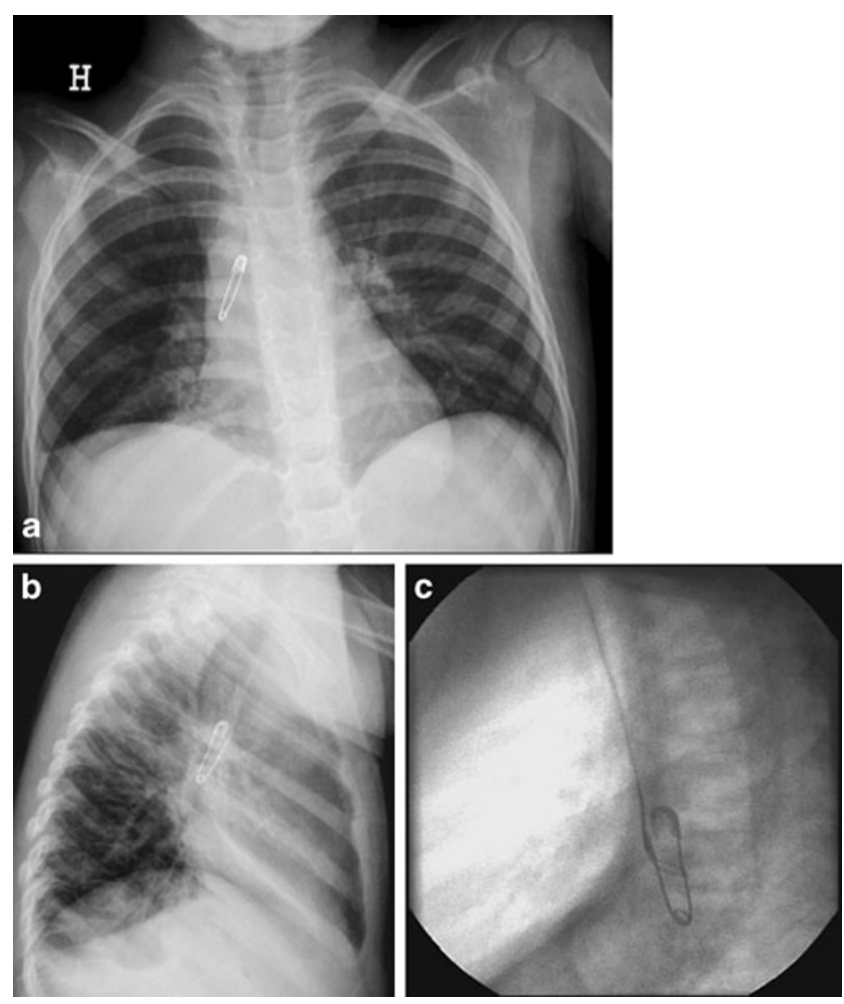

Fig. 10 a-c Foreign body in a 3-year-old girl with a 9-month history of coughing. a Chest radiography in the anteroposterior view and $\mathbf{b}$ the lateral view shows an open safety pin in the right main bronchus. $\mathbf{c}$ Removal of the pin with a snare during bronchography

\section{Asthma}

Five criteria are usually used to identify asthma in children:

1. Symptoms of recurrent coughing/wheezing

2. Other causes excluded

3. Risk factors of asthma identified

4. Improvement with asthma therapy

5. Confirmatory physical examination and/or diagnostic tests [31]

The children may present with hyper-inflated lungs and dramatic clinical symptoms with severe expiratory wheezing and respiratory distress. Asthma is not related to a special age group, but infants often have more severe symptoms because of the smaller calibre of the airways. The role of radiology is to recognise the possible acute complications of asthma that may require treatment. Chest radiography is used to demonstrate air leaks, especially pneumomediastinum, atelectasis or pneumonia. It is also important to rule out the other causes such as a foreign body or a viral infection or a really rare case that may simulate asthma clinically such as Kartagener's syndrome or haemosiderosis [32]. The longstanding complications of asthma (atelectasis/infection) demand further investigation with HRCT/MDCT, bronchoscopy with bronchoalveolar 
Fig. 11 a, b Foreign body in 1-year-old baby with acute wheezing. a Chest radiography with $\mathbf{b}$ the lateral view reveals a screw in the proximal part of the oesophagus and minor linear opacities in the lower part of the right lung
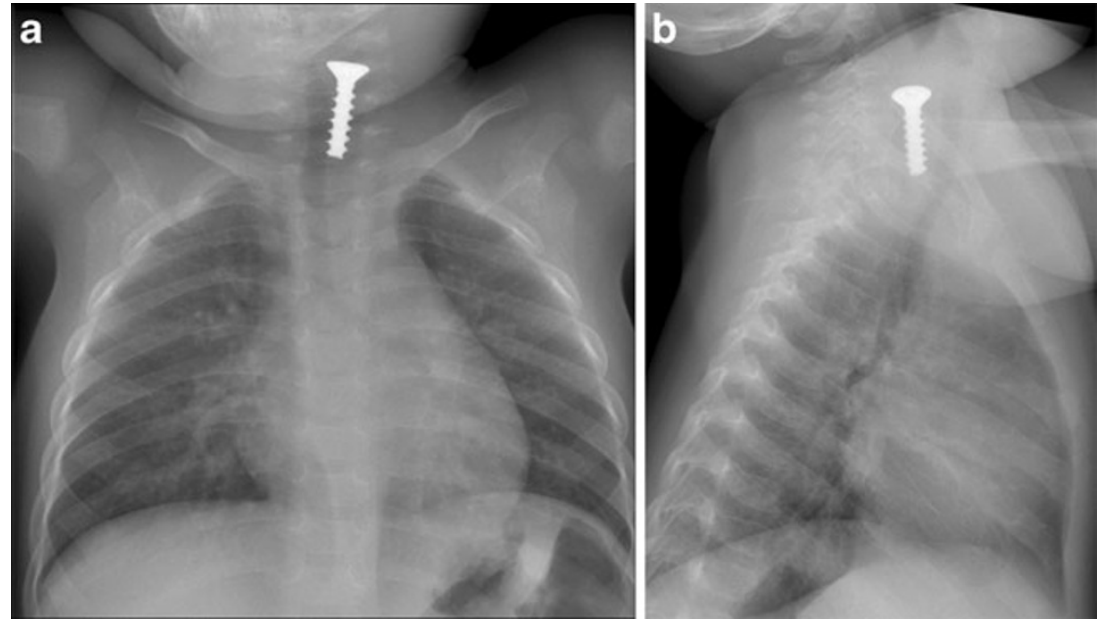

lavage and possibly bronchography in order to find underlying tracheobronchial anomalies and malacia.

\section{Congenital cystic lung malformations}

Congenital cystic lung disease consists of several entities that may be diagnosed antenatally but may also be detected after birth and during early childhood.

Congenital lobar emphysema Congenital lobar emphysema (CLE) most commonly involves the left upper or right middle lobe with compression of the bronchus causing a check-valve mechanism resulting in progressive hyperinflation of the affected lobe and the compression of adjacent lobes, the contralateral lung and mediastinal structures. Chest radiography is the primary examination, preferably in two planes. There is usually a gradual development of clinical and radiological signs with an often normal chest $\mathrm{X}$-ray at birth with increasing findings with emphysema in the following days (Fig. 12). Proposed aetiologies include focally deficient bronchial wall cartilage and connective tissue stroma resulting in abnormal support and obstruction of the affected bronchus. Contrast-enhanced CT is used in patients with persisting symptoms to rule out extrinsic vascular compression as an infrequent cause of obstruction or pneumothorax and to depict the anatomy of the affected lobe and degree of compression. If air trapping persists, surgical removal of the lobe is indicated to save the other compressed lobes of the lung $[33,34]$.

CCAM and BPS Congenital cystic adenomatoid malformation is a rare bronchopulmonary malformation located in the lung. It is most commonly unilobar with cystic and solid lesions with a normal vascular supply from the pulmonary artery. They may be hybrid lesions to BPS that consist of only solid lesions with blood supply from the aorta and no communication to the bronchial tree. Several classifications of CCAM have been proposed. The most frequently used, developed by Stocker et al. [35], describes four types of lesions depending on the size of the lesion and the presence of cystic and solid components: type 1 lesions present as single or multiple cysts $>2 \mathrm{~cm}$ in size, type 2 lesions are single or multiple cysts $<2 \mathrm{~cm}$ in size, type 3 are predominantly solid lesions with cysts $<0.5 \mathrm{~cm}$ and type 4
Fig. 12 a, b Congenital lobar emphysema in a term baby. a At day 1 of life the chest radiography is normal. b Three days later there are increasing respiratory problems, and radiography shows emphysema in the left upper lobe compressing the lower lobe and the right lung
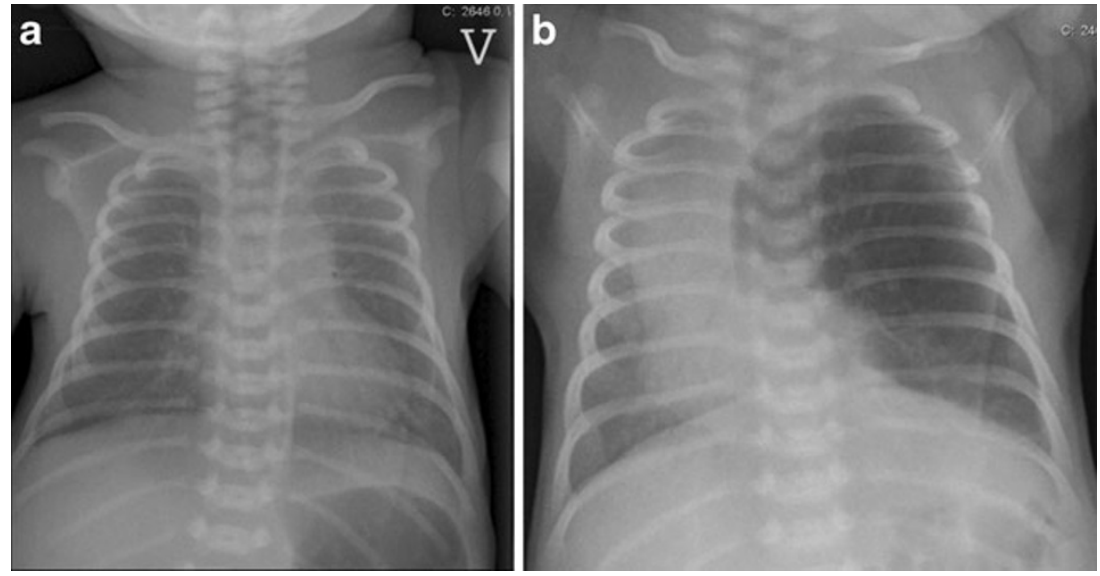
Fig. 13 a-d Vascular ring in a boy, 10 years of age, presenting with acute stridor when playing football. a Chest radiography with upper GI series, the anteroposterior view and $\mathbf{b}$ the lateral view shows a typical dorsal impression in the oesophagus suspected to be a vascular ring. c CTA, axial plane and d VR show a right-sided aortic arch with tracheal and oesophageal compression by a remaining fibrous ligament and a diverticulum of Kommerell (arrow)
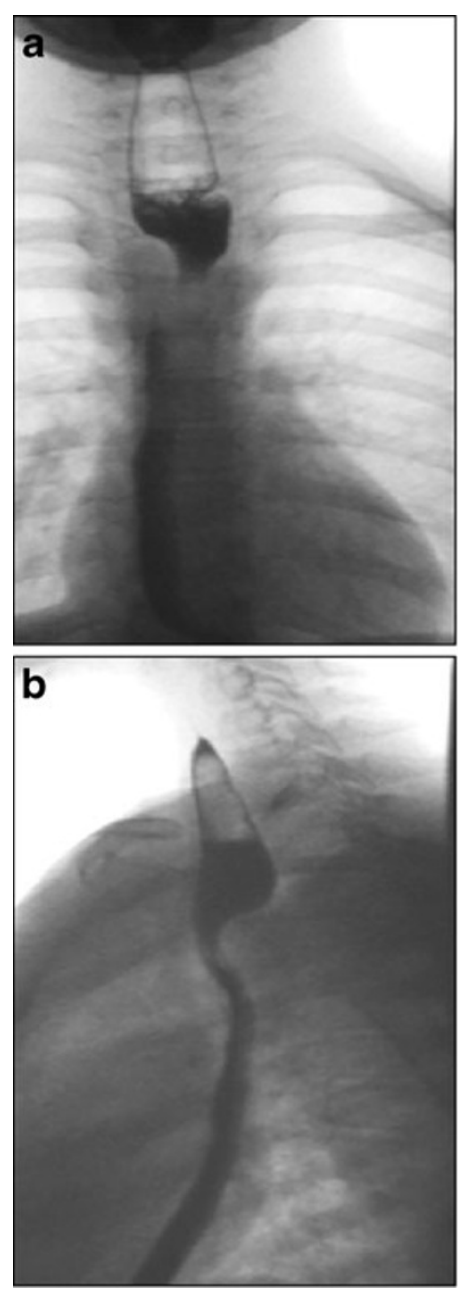
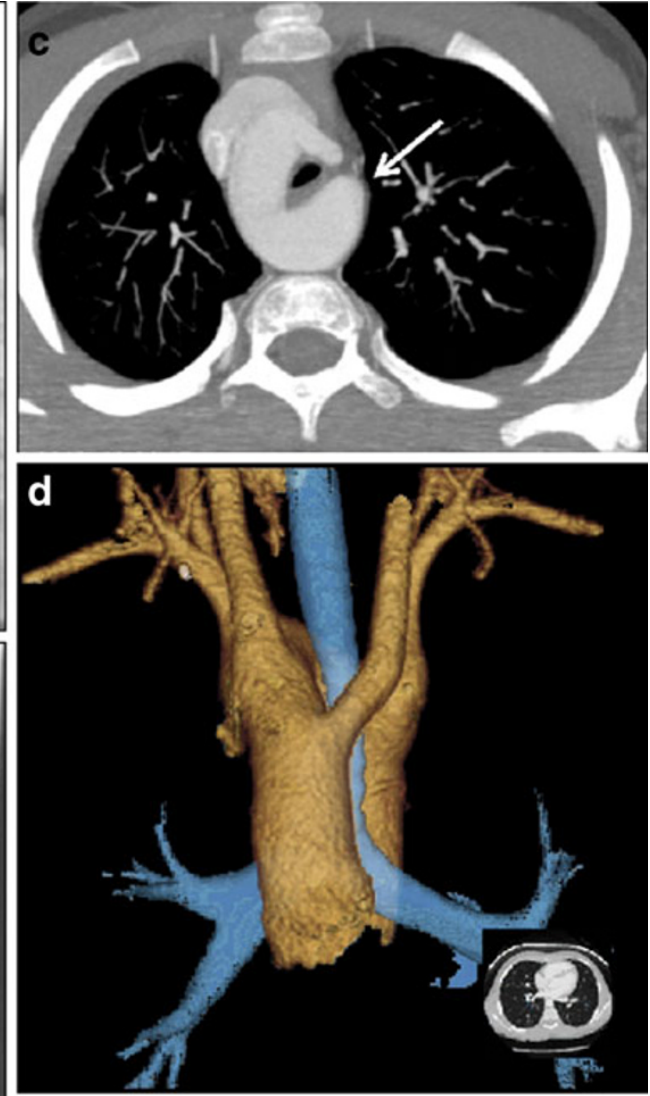

CCAM lesions are large peripheral thin-walled cysts. This classification can be difficult to use in some CCAMs and in hybrid lesions. A more simple classification differentiating the antenatally diagnosed CCAMs into only two types, macro- and microcystic lesions, has been proposed [36]. An anatomical classification, with a description of the nature of the lesion, arterial supply and venous drainage, is probably the most useful for radiological preoperative management. Most cases are diagnosed antenatally, and the smaller lesions do not usually present with respiratory problems. Larger lesions may present with respiratory failure needing urgent radiological evaluation with chest radiography and ideally CT. In postnatal asymptomatic cases, chest radiography is always performed but may underestimate the lesion. Contrast-enhanced CT/ CTA is required to rule out the presence of sequestration and to see the extent and aspect of the CCAM. Regression of small CCAMs may occur spontaneously, but some authors claim that there is the potential for malignancy to develop in the remaining lesions. Follow-up CT at the age of 1 month and later surgical removal of the affected lobe are recommended by some institutions. However, the timing of the follow-up and treatment of CCAM remains controversial, and surgical treatment is not always performed [37]. BPS is treated with embolisation or surgical excision.

Vascular rings Vascular rings and slings are also among the congenital anomalies that may cause acute symptoms (stridor, cough and cyanotic episodes). When compression of the neighbouring organs becomes significant, swallowing or feeding problems may occur. Vascular rings can be identified with upper GI series and echocardiography. The contours of the oesophagus may be suggestive of a vascular ring, and further examinations with CTA/MRA should then be performed. Cross-sectional imaging with MPR and 3D VR reconstructions is helpful in clarifying the anatomy and facilitates surgical planning before thoracotomy. In newborn babies and smaller children, CTA is often preferred over MRA, despite the exposure to radiation, because of higher spatial resolution of the small calibre vessels and airways and no need for general anaesthesia or sedation. When the evaluation of the congenital cardiopathy associated with respiratory failure is warranted, CTA is preferred in smaller 
Fig. 14 a-c Boy, 11 years of age, with a 2-month history of cough, weight loss and lymph nodes in the neck. a Chest radiography shows a large mediastinal mass affecting the apex of the right hemithorax with severe tracheal compression. b Coronal plane of a contrast-enhanced CT with a better delineation of the tumour and airway compression of both main bronchi. c The corresponding coronal fluorodeoxy-glucose (FDG) PET-CT, with pathological uptake of FDG of active disease extending up to the right side of the neck
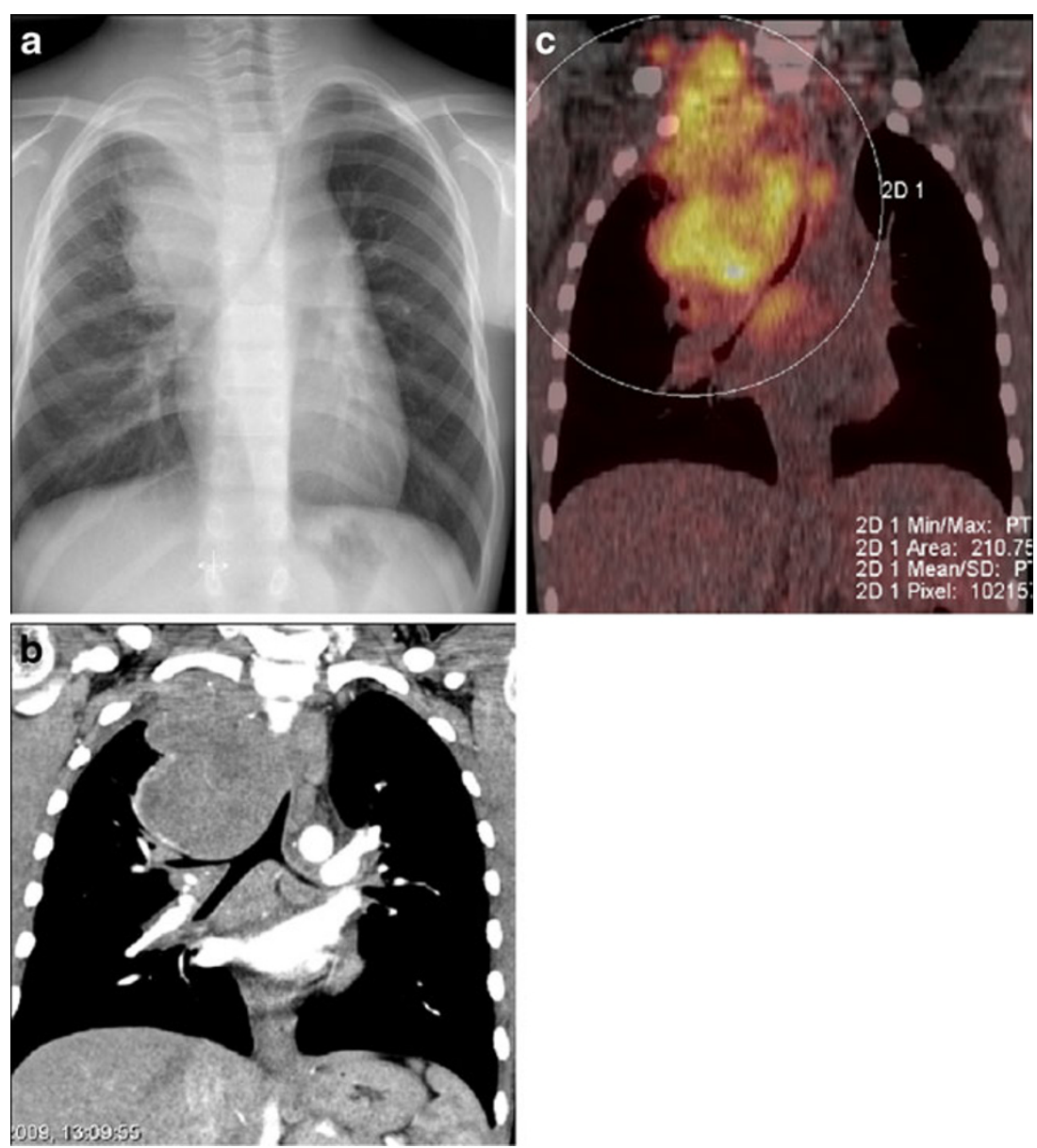

children. Often mechanical compression of the main airways may cause obstructive emphysema, atelectasis needing surgical repair. In infants and older children, MR/MRA can sufficiently depict vascular and airway anatomy and should be performed when available. The most common vascular ring is the combination of double aortic arch or a right aortic arch and an aberrant left subclavian artery with the ductus arteriosus or ductal remnant completing the ring (Fig. 13).

\section{Congenital abnormalities-fibromatosis/vascular malformations}

Various forms of vascular malformations and fibromatosis are the most common soft tissue expansions in children. They frequently present in the region of the neck but can extend to the chest wall and thoracic cavity. Acute symptoms may occur if the mass is compressing the airways. Ultrasound with colour- and spectral Doppler can evaluate blood flow while MRI with MRA can demonstrate the total extent of the lesion. Angiography is performed when combined with interventional treatment for arteriovenous malformations.

\section{Acquired abnormalities - thoracic tumours}

Tumours may occur in the anterior, middle or posterior part of the mediastinum. Substantial narrowing of the trachea or major bronchi by a mass can occur without significant compromise of breathing at rest, and the calibre of the airway should be determined with radiography and $\mathrm{CT}$. It is important to evaluate the risk of life-threatening airway obstruction before any procedure requiring general anaesthesia or heavy sedation in a patient with a large anterior mediastinal mass (Fig. 14). Ultrasound may be used for the diagnosis of lesions near the thoracic wall. MRI and possibly PET-CT may be needed in the staging and follow-up of some diseases, especially lymphoma [37, 38].

Other neoplasms that can narrow the airway by extrinsic mass effect include infantile haemangioma, rhabdomyosarcomas, neurogenic tumours, germ cell tumours and, rarely, thyroid neoplasms [39]. Neuroblastoma/ganglioneuroblastoma/ganglioneuroma may arise where sympathic tissue exists and may be seen in the neck, posterior mediastinum, adrenal gland, retroperitoneum and pelvis. The three tumours differ in their degree of cellular and extracellular maturation; immature tumours tend to be aggressive and 
occur in younger patients (median age, just under 2 years), whereas mature tumours occur in older children (median age, approximately 7 years) and tend to be more benign. The symptoms vary but are often dominated by pain and neurological symptoms. Acute compression of the spinal cord by a tumour requires rapid neurosurgical intervention.

\section{Conclusion}

There are many different causes of thoracic emergencies in children, some specific to a certain age group. A wide variety of different conditions may lead to thoracic emergencies in children. The most urgent and important ones involve the airways and may be life-threatening. Radiology has become increasingly important in the acute management of these emergencies. Radiography is still the most important tool and can be supplemented with ultrasound, CT and MRI. A skilled radiologist familiar with paediatric pathological conditions plays an important role in helping clinicians to ascertain the diagnosis and to start adequate treatment.

\section{References}

1. Donnelly LF (2005) Imaging in immunocompetent children who have pneumonia. Radiol Clin North Am 43:253-265

2. Wildin SR, Chonmaitree T, Swischuk LE (1988) Roentgenographic features of common pediatric viral respiratory tract infections. Am J Dis Child 142:43-46

3. Breysem L, Loyen S, Boets A, Proesmans M, De BK, Smet MH (2002) Pediatric emergencies: thoracic emergencies. Eur Radiol 12:2849-2865

4. Hall EJ (2009) Radiation biology for pediatric radiologists. Pediatr Radiol 39(Suppl 1):S57-S64

5. Frush DP, Frush KS (2008) The ALARA concept in pediatric imaging: building bridges between radiology and emergency medicine: consensus conference on imaging safety and quality for children in the emergency setting, Feb. 23-24, 2008, Orlando, FL-executive summary. Pediatr Radiol 38(Suppl 4): S629-S632

6. Goske MJ, Applegate KE, Boylan J, Butler PF, Callahan MJ, Coley BD, Farley S, Frush DP, Hernanz-Schulman M, Jaramillo D, Johnson ND, Kaste SC, Morrison G, Strauss KJ, Tuggle N (2008) The 'Image Gently' campaign: increasing CT radiation dose awareness through a national education and awareness program. Pediatr Radiol 38:265-269

7. Brody AS, Frush DP, Huda W, Brent RL (2007) Radiation risk to children from computed tomography. Pediatrics 120:677-682

8. Smith J, Schumacher RE, Donn SM, Sarkar S (2011) Clinical course of symptomatic spontaneous pneumothorax in term and late preterm newborns: report from a large cohort. Am J Perinatol 28:163-168

9. Lawal TA, Gluer S, Reismann M, Dordelmann M, Schirg E, Ure B (2009) Spontaneous neonatal pneumomediastinum: the "spinnaker sail" sign. Eur J Pediatr Surg 19:50-52

10. Nissen MD (2007) Congenital and neonatal pneumonia. Paediatr Respir Rev 8:195-203
11. Lee T, Lee SK (1997) Upper airway obstruction in infants and children: evaluation by tracheobronchography with a non-ionic contrast agent. Pediatr Radiol 27:276-280

12. MacIntyre P, Peacock C, Gordon I, Mok Q (1998) Use of tracheobronchography as a diagnostic tool in ventilatordependent infants. Crit Care Med 26:755-759

13. McLaren CA, Elliott MJ, Roebuck DJ (2005) Tracheobronchial intervention in children. Eur J Radiol 53:22-34

14. Lee KS, Boiselle PM (2010) Update on multidetector computed tomography imaging of the airways. J Thorac Imaging 25:112124

15. Taylor GA, Atalabi OM, Estroff JA (2009) Imaging of congenital diaphragmatic hernias. Pediatr Radiol 39:1-16

16. Baglaj M, Dorobisz U (2005) Late-presenting congenital diaphragmatic hernia in children: a literature review. Pediatr Radiol 35:478-488

17. Betremieux P, Dabadie A, Chapuis M, Pladys P, Treguier C, Fremond B, Lefrancois C (1995) Late presenting Bochdalek hernia containing colon: misdiagnosis risk. Eur J Pediatr Surg 5:113-115

18. Trachsel D, Selvadurai H, Bohn D, Langer JC, Coates AL (2005) Long-term pulmonary morbidity in survivors of congenital diaphragmatic hernia. Pediatr Pulmonol 39(5):433-439

19. Durand C, Piolat C, Nugues F, Bessaguet S, Alvarez C, Baudain $P$ (2005) Emergency pediatric thoracic radiology. J Radiol 86(2 Pt 2):198-206

20. Kim YW, Donnelly LF (2007) Round pneumonia: imaging findings in a large series of children. Pediatr Radiol 37:1235-1240

21. Heulitt MJ, Ablow RC, Santos CC, O'Shea TM, Hilfer CL (1988) Febrile infants less than 3 months old: value of chest radiography. Radiology 167:135-137

22. Alario AJ, McCarthy PL, Markowitz R, Kornguth P, Rosenfield N, Leventhal JM (1987) Usefulness of chest radiographs in children with acute lower respiratory tract disease. J Pediatr 111:187-193

23. Ellis JR, Gleeson FV (2002) Non-traumatic thoracic emergencies: imaging and treatment of thoracic fluid collections (including pneumothorax). Eur Radiol 12:1922-1930

24. Calder A, Owens CM (2009) Imaging of parapneumonic pleural effusions and empyema in children. Pediatr Radiol 39:527-537

25. Proesmans M, De BK (2009) Clinical practice: treatment of childhood empyema. Eur J Pediatr 168:639-645

26. Donnelly LF, Klosterman LA (1998) Cavitary necrosis complicating pneumonia in children: sequential findings on chest radiography. AJR Am J Roentgenol 171(1):253-256

27. Balfour-Lynn IM, Abrahamson E, Cohen G, Hartley J, King S, Parikh D, Spencer D, Thomson AH, Urquhart D (2005) BTS guidelines for the management of pleural infection in children. Thorax 60(Suppl 1):i1-i21

28. Silva AB, Muntz HR, Clary R (1998) Utility of conventional radiography in the diagnosis and management of pediatric airway foreign bodies. Ann Otol Rhinol Laryngol 107(10 Pt 1):834-848

29. Tahir N, Ramsden WH, Stringer MD (2009) Tracheobronchial anatomy and the distribution of inhaled foreign bodies in children. Eur J Pediatr 168:289-295

30. Karakoc F, Karadag B, Akbenlioglu C, Ersu R, Yildizeli B, Yuksel M, Dagli E (2002) Foreign body aspiration: what is the outcome? Pediatr Pulmonol 34:30-36

31. Liu A, Covar R (2008) Asthma/clinical features, outcomes, and prognosis. In: Taussig LM, Landau LI (eds) Pediatric respiratory medicine, 2nd ed. Mosby Elsevier, Philadelphia, p 806

32. Ioachimescu OC, Sieber S, Kotch A (2004) Idiopathic pulmonary haemosiderosis revisited. Eur Respir J 24:162-170

33. Tempe DK, Virmani S, Javetkar S, Banerjee A, Puri SK, Datt V (2010) Congenital lobar emphysema: pitfalls and management. Ann Card Anaesth 13:53-58 
34. Ulku R, Onat S, Ozcelik C (2008) Congenital lobar emphysema: differential diagnosis and therapeutic approach. Pediatr Int 50:658-661

35. Stocker JT, Madewell JE, Drake RM (1977) Congenital cystic adenomatoid malformation of the lung. Classification and morphologic spectrum. Hum Pathol 8:155-171

36. Cha I, Adzick NS, Harrison MR, Finkbeiner WE (1997) Fetal congenital cystic adenomatoid malformations of the lung: a clinicopathologic study of eleven cases. Am J Surg Pathol 21:537-544
37. Lakhoo K (2009) Management of congenital cystic adenomatous malformations of the lung. Arch Dis Child Fetal Neonatal Ed 94: F73-F76

38. Toma P, Granata C, Rossi A, Garaventa A (2007) Multimodality imaging of Hodgkin disease and non-Hodgkin lymphomas in children. Radiographics 27:1335-1354

39. Lonergan GJ, Schwab CM, Suarez ES, Carlson CL (2002) Neuroblastoma, ganglioneuroblastoma, and ganglioneuroma: radiologic-pathologic correlation. Radiographics 22:911-934 